Reprod. Nutr. Dévelop., 1986, 26 (1 B), 281-282.

\title{
Adaptation comportementale à l'alimentation sèche des chevrettes pendant le sevrage
}

\author{
P. MORAND-FEHR, J. HERVIEU, A. FAYE, M. ROUASSI
}

Station de Nutrition et Alimentation, I.N.R.A., Institut National Agronomique Paris-Grignon 16, rue Claude-Bernard, 75231 Paris Cedex 05.

Summary. The feeding behaviour of 12 young Alpine and Saanen female goats reared in a group was observed from 2 days before to 14 days after weaning. The duration of daily ingestion and behaviour during a meal were quickly the same as in adult goats, but the time needed to eat hay was longer and the number of meals eaten was higher than in adults.

L'étude du comportement alimentaire du chevreau au cours du sevrage a pour but de préciser la notion de repas pendant cette période et les principaux paramètres de la prise alimentaire d'un jour avant à 15 jours après le sevrage. Elle fait suite à celle de la chèvre adulte (Morand-Fehr et al., 1980).

Matériel et méthodes. Douze chevrettes Alpine et Saanen élevées dans un enclos paillé de $8,4 \mathrm{~m}^{2}$ ont été sevrées à un âge moyen de 49,1 jours. Avant le sevrage, elles reçoivent à volonté du lait de remplacement par un allaiteur automatique, du foin de luzerne de bonne qualité et un mélange granulé d'aliments concentrés ; elles disposent d'eau et d'une pierre à sel. Le jour du sevrage, le lait est supprimé brutalement. Le foin et l'aliment concentré sont renouvelés 4 fois par jour à $7,11,14$ et $17 \mathrm{~h}$ et la litière, chaque jour à $11 \mathrm{~h}$. L'éclairement des animaux est maintenu $12 \mathrm{~h}$ par jour (7-19 h). Le comportement alimentaire de 6 chevrettes du lot est enregistré par contrôle visuel chaque minute de 7 à $17 \mathrm{~h}$ deux jours par semaine.

Résultats et discussion. Les chevreaux consomment toujours plus de foin que d'aliment concentré (tabl. 1). La consommation de foin augmente rapidement pendant les 4 jours $(S+4)$ qui suivent le sevrage et se stabilise à partir du $7^{\text {e }}$ jour $(S+7)$. La capacité volumique du rumen est déjà peut-être atteinte. En revanche, l'ingestion d'aliment concentré augmente nettement jusqu'à $S+14$.

Les chevrettes semblent avoir besoin d'un délai de 2 semaines pour consommer les aliments secs plus rapidement. Toutefois, leur vitesse d'ingestion de foin est encore à $S+14$, inférieure d'environ $40 \%$ à celles des adultes (Morand-Fehr et al., 1980). La durée totale d'ingestion par jour est voisine de celle des adultes (à $10 \%$ près) dès $S+4$.

Le repas du chevreau a été défini comme l'ensemble de prises alimentaires espacées de moins de $10 \mathrm{~min}$ puisque si l'intervalle entre 2 prises varie de 5 à 25 min, le nombre de repas par jour reste identique et que cette définition coïncide avec celles des chèvres adultes. 
Entre 7 et $17 \mathrm{~h}$, le nombre de repas est peu variable de $S-1$ à $S+14$, mais nettement supérieur à celui des adultes. Toutefois, il est certainement influencé par le nombre élevé de distributions d'aliments. La durée des repas est relativement faible mais augmente dès que la quantité de foin ingéré s'élève. Même avant le sevrage, la consommation de foin a une position importante au cours du repas puisqu'elle prend toujours, même juste avant le sevrage, plus de temps que la consommation des autres aliments et que les prises alimentaires de foin pendant un repas sont plus fréquentes que celles de paille, de concentrés ou d'eau.

TABL. 1. - Evolution des paramètres caractérisant le comportement alimentaire au cours du sevrage du chevreau.

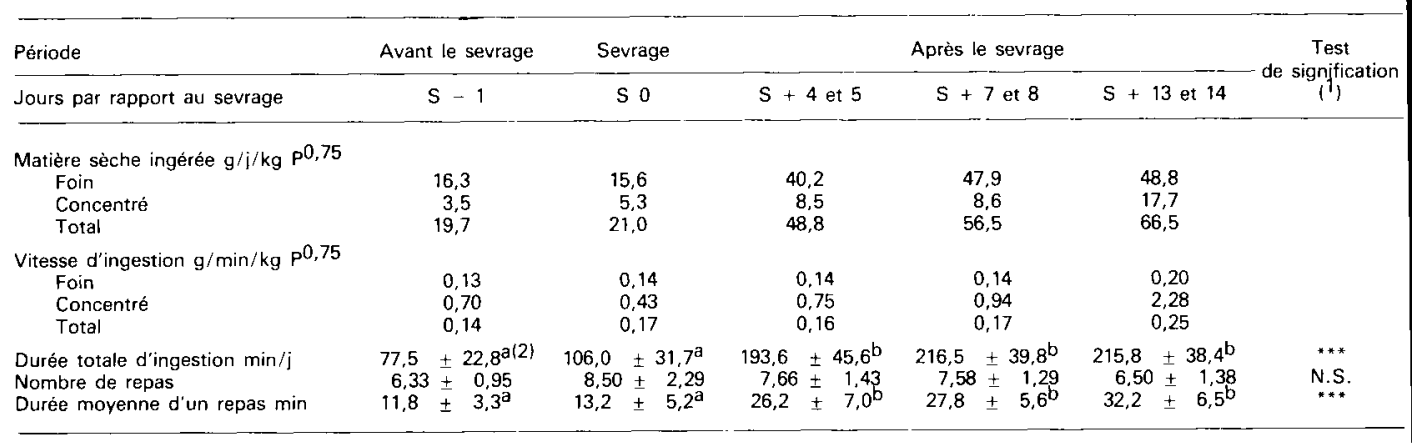

$(1): * * P \leq 0,001 ; N . S$. Non significatif. $(2)$ : Les valeurs n'étant pas affectées de la même lettre sont significativement différentes au seuil de $P \leq 0,01$.

En conclusion, à part le jour du sevrage nous pouvons considérer que la chevrette a très rapidement un comportement alimentaire cohérent et proche de l'adulte (Morand-Fehr et al., 1980) dès 2 semaines après le sevrage et même avant le sevrage pour certains aspects comme le déroulement du repas.

Morand-Fehr P., Hervieu J., Sauvant D., 1980. Contribution à la description de la prise alimentaire de la chèvre. Reprod. Nutr. Dévelop., 20, 1641-1644. 